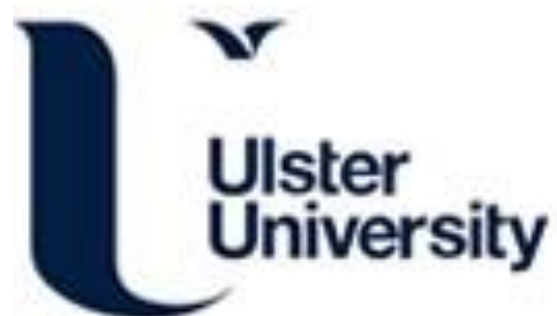

\section{Carotenoids and co-antioxidants in age-related maculopathy: design and methods.}

Neelam, K., Hogg, RE., Stevenson, MR., Johnston, E., Anderson, R., Beatty, S., \& Chakravarthy, U. (2008). Carotenoids and co-antioxidants in age-related maculopathy: design and methods. Ophthalmic Epidemiology, $15,389-401$

Link to publication record in Ulster University Research Portal

\section{Published in:}

Ophthalmic Epidemiology

Publication Status:

Published (in print/issue): 01/01/2008

\section{Document Version}

Publisher's PDF, also known as Version of record

\section{General rights}

Copyright for the publications made accessible via Ulster University's Research Portal is retained by the author(s) and / or other copyright owners and it is a condition of accessing these publications that users recognise and abide by the legal requirements associated with these rights.

\section{Take down policy}

The Research Portal is Ulster University's institutional repository that provides access to Ulster's research outputs. Every effort has been made to ensure that content in the Research Portal does not infringe any person's rights, or applicable UK laws. If you discover content in the Research Portal that you believe breaches copyright or violates any law, please contact pure-support@ulster.ac.uk. 


\title{
Carotenoids and Co-Antioxidants in Age-Related Maculopathy: Design and Methods
}

\author{
Kumari Neelam, ${ }^{1}$ Ruth E. Hogg, ${ }^{2}$ Michael R. Stevenson, ${ }^{2}$ Elinor Johnston, ${ }^{2}$ Roger Anderson, ${ }^{1,3}$ Stephen Beatty, ${ }^{2}$ \\ and Usha Chakravarthy ${ }^{2,4}$ \\ ${ }^{1}$ Waterford Institute of Technology, Waterford, Republic of Ireland \\ and Waterford Regional Hospital, Waterford, Republic of Ireland \\ ${ }^{2}$ The Queen's University, Belfast, United Kingdom \\ ${ }^{3}$ University of Ulster at Coleraine, Northern Ireland, United Kingdom \\ ${ }^{4}$ Royal Victoria Hospitals, Belfast, United Kingdom
}

\begin{abstract}
Age-related macular degeneration (AMD), is the leading cause of blind registration in the Western World among individuals 65 years or older. Early AMD, a clinical state without overt functional loss, is said to be present clinically when yellowish deposits known as drusen and/or alterations of fundus pigmentation are seen in the macular retina. Although the etiopathogenesis of AMD remains uncertain, there is a growing body of evidence in support of the view that cumulative oxidative damage plays a causal role. Appropriate dietary antioxidant supplementation is likely to be beneficial in maintaining visual function in patients with AMD, and preventing or delaying the progression of early AMD to late AMD. The Carotenoids in Age-Related Maculopathy (CARMA) Study is a randomized and double-masked clinical trial of antioxidant supplementation versus placebo in $\mathbf{4 3 3}$ participants with either early AMD features of sufficient severity in at least one eye or any level of AMD in one eye with late AMD (neovascular AMD or central geographic atrophy) in the fellow eye. The aim of the CARMA Study is to investigate whether lutein and zeaxanthin, in combination with co-antioxidants (vitamin C, E, and zinc), has a beneficial effect on visual function and/or prevention of progression from early to late stages of disease. The primary outcome is improved or preserved distance visual acuity at 12 months. Secondary outcomes include improved or preserved interferometric acuity, contrast sensitivity, shape discrimination ability, and change in AMD severity as monitored by fundus photography. This article outlines the CARMA Study design and methodology, including its rationale.
\end{abstract}

\section{INTRODUCTION}

Age-related macular degeneration (AMD), previously also known as age-related maculopathy, is the term used to describe a spectrum of ageing changes in the macular retina of the eyes of older adults. In the early stages a large proportion of older adults have yellowish deposits known as drusen and alterations of fundus pigmentation in the macular retina. This is known as early

Received 8 April 2007; accepted 31 January 2008

Keywords: Age-Related maculopathy; antioxidant

supplementation; interferometric acuity; macular pigment;

oxidative damage; randomized clinical trial

Correspondence to:

Prof. Usha Chakravarthy

Ophthalmology and Vision Science

Queen's University and Royal Victoria Hospitals

Belfast BT 12 6BA, United Kingdom.

email: U.Chakravarthy@queens-belfast.ac.uk
AMD. In a significant proportion of eyes exhibiting early AMD there is progression through intermediate (more severe levels of drusen and pigmentary irregularities) to late stages with dysfunction and death of photoreceptors secondary to an atrophic (geographic atrophy, GA) and/or a neovascular (choroidal neovascularization, CNV) event. These late stages are commonly referred to as late AMD. Currently, late AMD is the leading cause of blind registration in Western countries among individuals 65 years and older, ${ }^{1-3}$ with $\mathrm{CNV}$ accounting for $90 \%$ of these cases. ${ }^{4,5}$

In the future, the prevalence and incidence of AMD is expected to rise because of an increase in life expectancy, and the consequential demographic shift towards an elderly population will have implications for the socio-economic impact of this disease ${ }^{6,7}$ Currently available therapeutic interventions for this condition are largely aimed at preservation of presenting visual acuity (VA) ${ }^{8-11}$ Although a significant proportion of patients may achieve some visual gain with the novel inhibitors of vascular endothelial growth factor, complete restoration of 
discriminant visual function is only achieved in small proportions of those treated. ${ }^{12,13}$ Moreover, there is no available treatment for atrophic AMD. There is no doubt that effect of late AMD on the activities of daily living, self-care, emotional well being, and overall quality of life are important, and that these are related to disease severity. ${ }^{14-19}$ Therefore, prevention or delay of early to late manifestations represents the best means of limiting the impact of this degenerative disorder on visual health and quality of life in the elderly population.

While the exact etiopathogenesis of the disorder remains uncertain, there is a growing body of evidence that cumulative oxidative damage may play a causal role. ${ }^{20,21}$ Oxidative damage refers to tissue injury by reactive oxygen intermediates, which are unstable molecules generated in cells as a by-product of oxygen metabolism, and, additionally, in the retina, by photochemical reactions between light and oxygen. Consequently, antioxidant supplementation could play an important role in preventing the development of $\mathrm{AMD}$, or delaying the progression from early to late disease.

\section{STUDY RATIONALE}

One of the unique features of the primate macula is the presence of high concentrations of the xanthophylls, lutein (L) and zeaxanthin (Z), which gives it its eponymous yellow color. Together, these two xanthophylls are referred to as macular pigment (MP). ${ }^{22}$ MP peaks in the central 1 to 2 degrees of the fovea, and declines to optically negligible levels by 5 to 10 degrees radial eccentricity. ${ }^{23}$ Of note, MP cannot be synthesized de novo, and is derived entirely from diet.

Macular pigment is purported to prevent or retard the development or progression of AMD because of its ability to absorb blue light at the prereceptorial level, and because of its capacity to quench reactive oxygen intermediates via its powerful antioxidant activity. ${ }^{20,21}$ Furthermore, it has been observed that increased intake of dietary $\mathrm{L}$ and $\mathrm{Z}$ results in the augmentation of MP ${ }^{24}$ consistent with the possibility that appropriate dietary supplementation or modification would confer protection against development and/or progression of AMD.

The Age-Related Eye Disease Study (AREDS) has investigated the role of multiple antioxidants, namely vitamins A,C, and $\mathrm{E}$ and zinc, and demonstrated that treatment reduces the relative risk of disease progression to late AMD by $25 \%$ and results in odds reduction of $27 \%$ for visual acuity loss. ${ }^{25}$ These beneficial effects were mainly observed in the fellow eyes of a subset of participants who had late stage AMD in the contralateral eye. Noteworthy, too, was the protective effect in those participants with intermediate stages of AMD in both eyes.

To date, five interventional studies have investigated the role of $\mathrm{L}$ supplementation in AMD patients (Table 1), ${ }^{26-30}$ and the data from four of these demonstrated improvements in acuity and/or other parameters of visual function in the treatment groups. ${ }^{27-30}$ Although the statistical power of these studies is limited by their small sample sizes $(\mathrm{n} \leq 90)$, they are, nonetheless, encouraging. More recently Chong and colleagues undertook a systematic review and meta-analysis of dietary antioxidants and supplements, and questioned the value of primary prevention. ${ }^{31}$ However, the findings of AREDS in terms of secondary prevention, particularly in the fellow eyes of patients with advanced disease in the contralateral eye, raises intriguing questions on the potential benefits of supplemental antioxidants.

Furthermore, a number of well-conducted population-based studies employing longitudinal study design have suggested that high levels of dietary antioxidants, specifically $\mathrm{L}$ and $\mathrm{Z}$, may have protective and beneficial effects in retarding progression to advanced AMD (Table 2). Lutein and $\mathrm{Z}$ were not included in the AREDS formulation because they were not commercially available in supplement form at the inception of that study. Further,

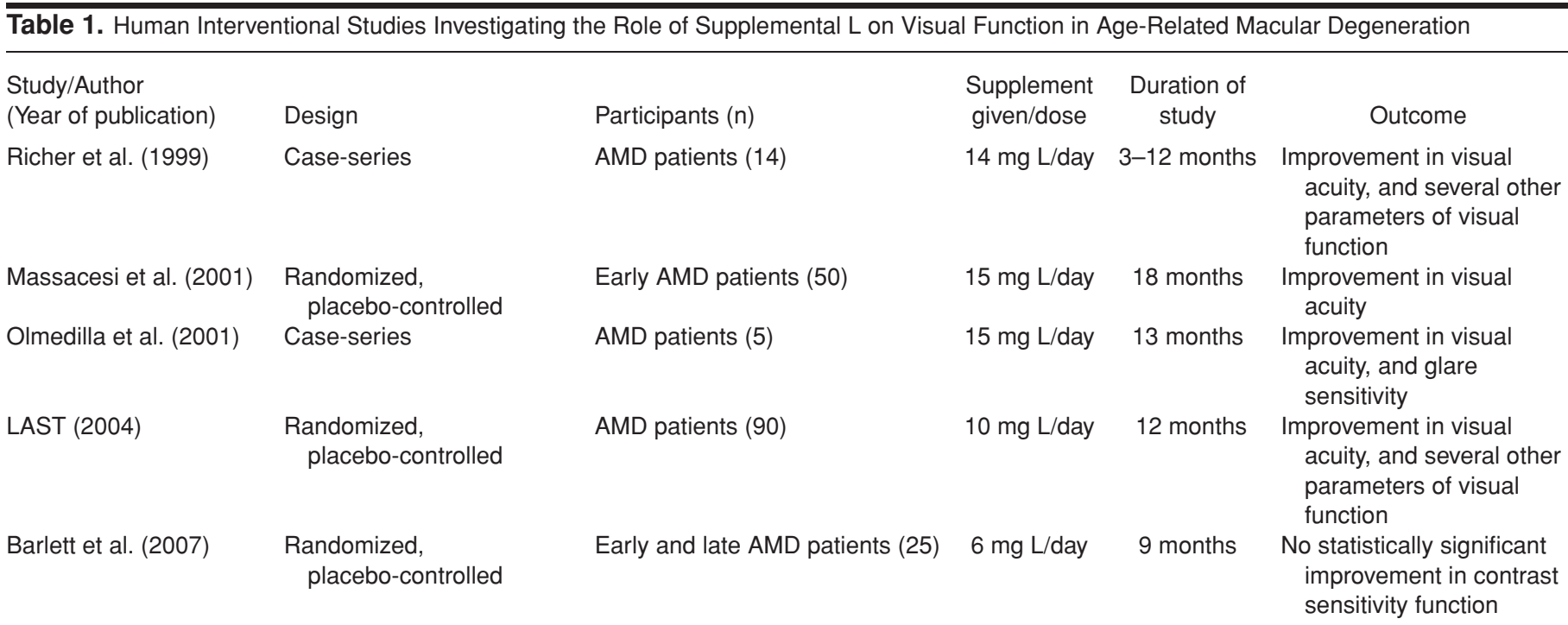

LAST: Lutein Antioxidant Supplementation Trial. L: Lutein. 


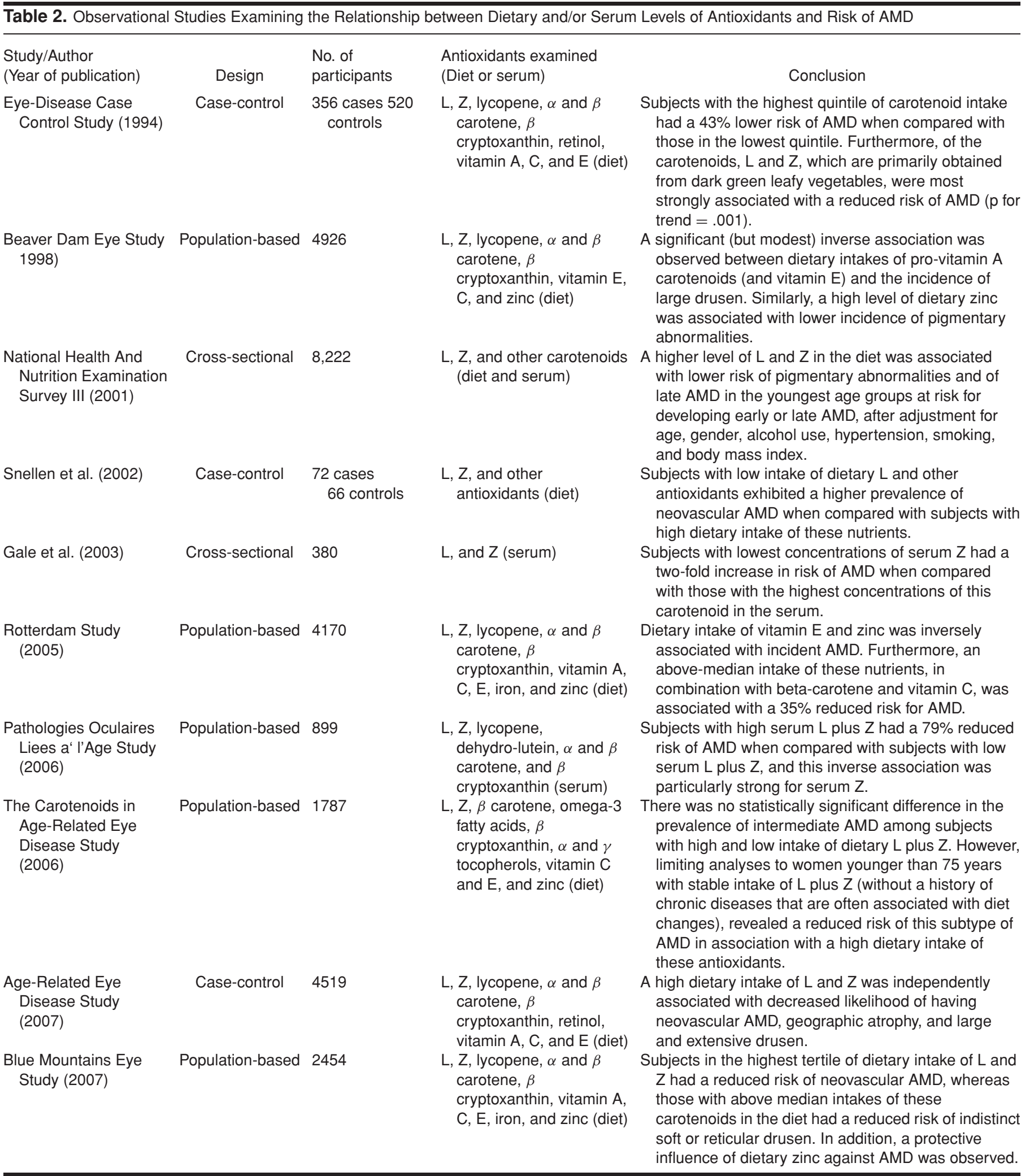


AREDS did not measure subtle differences in visual function between the various intervention groups following antioxidant supplementation. Thus there was an obvious need for controlled clinical trials with $\mathrm{L}$ and $\mathrm{Z}$ included in the test formulation. We hypothesized that reduction of oxidative stress at the level of the RPE could potentially improve function. It was our view that the detection of differences in visual function between intervention groups would provide both a better understanding of the pathogenesis, and add to the evidence base for the use of antioxidants in preventing progression of early or intermediate to late AMD.

Therefore, the Carotenoids in Age-Related Maculopathy (CARMA) Study, a randomized controlled clinical trial, was developed to test the hypothesis that a combination of antioxidant vitamins, including $\mathrm{L}$ and $\mathrm{Z}$, would be beneficial in maintaining visual function and reducing the risk of progression to a more severe morphological state. The rationale for adding $\mathrm{L}$ and $\mathrm{Z}$ to the antioxidants (already proven to have a beneficial effect in the AREDS Study) are two-fold: first, $\mathrm{L}$ and $\mathrm{Z}$ exhibit the ideal anatomic, biochemical, and optical properties to limit (photo) oxidative damage at the macula; second, carotenoids act synergistically with other antioxidants to exert antioxidant effect. ${ }^{32}$

\section{STUDY DESIGN}

The CARMA Study is a randomized and double-masked clinical trial of antioxidant supplementation versus placebo in 433 Caucasian AMD participants aged 50 years and above, who are recruited at two clinical centers in Ireland (Waterford and Belfast). Participants must have either early AMD features of sufficient severity in at least one eye or any level of AMD in one eye and advanced AMD (neovascular AMD or central GA) in the fellow eye. All enrolled participants will be followed until the last participant has completed one year of follow-up or until the Data and Safety Monitoring Committee (DSMC) has recommended earlier termination of the trial.

An overview of the administrating and coordinating infrastructures of the CARMA Study is given in Figure 1. The study is approved by the Waterford Research Ethics Committee, and by the Office for Research Ethics Committees in Northern Ireland. The CARMA Study is registered with a clinical trial database (International Standard Randomized Controlled Trial Number: 9455760).

\section{AIMS OF THE STUDY}

The principal aim of the CARMA Study is to investigate the potential efficacy of $\mathrm{L}$ and $\mathrm{Z}$, in combination with key vitamins and minerals, on psychophysical measures of vision namely distance visual acuity (DVA), contrast sensitivity, photopic interferometric acuity, and shape discrimination. Secondary aims are to examine the potential efficacy of the CARMA preparation in delaying or retarding the morphological progression of early AMD. The CARMA Study will also measure MP levels, and serum concentrations of $\mathrm{L}$ and $\mathrm{Z}$, in response to supplementation with the macular carotenoids.

\section{SAMPLE SIZE AND POWER CONSIDERATION}

It is hypothesized that treatment with CARMA preparation will result in either maintained function or improved function while treatment with placebo will result in decreasing function. The original study design employed photopic interferometric acuity as the primary outcome variable. However, we encountered several problems in the consistency and reliability of this measurement technique, and, therefore, following the DSMC recommendations during its meeting in October 2005, the primary outcome measure was changed from photopic interferometry to DVA. The study sample size was also increased in order to take into account the change in the primary outcome measure. The revised protocol was approved by the Research Ethics Committee at both the clinical centers.

The revised sample size calculations estimated that a sample size of 500 participants with 5\% alpha ( 2 sided test) will have $90 \%$ power to detect a 0.04 (2 logMAR letters) difference in DVA assuming that standard deviation of the change in DVA within a participant is 0.13 (6.5 logMAR letters) and that there would be a $10 \%$ attrition rate. The study duration was extended as a result of increase in the sample size.

\section{RECRUITMENT STRATEGIES}

Study awareness campaigns consisting of the distribution of leaflets and posters along with presentations describing CARMA are conducted at both sites. Potential participants are sent a letter of invitation along with the study information leaflet (explaining the background and rationale for the CARMA Study) containing contact details of the study staff. No financial incentives are offered to the participants; ${ }^{33}$ however, costs of transportation to and from the study center are provided, where requested.

All potential participants who expressed a willingness and desire to participate in the CARMA Study are screened at a study clinic where informed consent is obtained. Following verification of eligibility (Table 3), all participants are randomized to one of the two groups. The CARMA preparation is provided in numbered containers and the participant is instructed to take one tablet twice daily. The first participant in the CARMA Study was enrolled in July 2004, and new recruitments continued until April 2007 to achieve the revised sample size. All enrolled participants will be followed until the last participant has completed 1 year of follow-up, and, therefore, the study concluded on April 2008. Also, participants who are recruited early on will have the opportunity to be followed up for a maximum of 36 months.

The baseline/screening visit consists of four separate parts (informed consent, clinical examination, questionnaire, and randomization), and takes approximately $2-3$ hours per participant. The study is conducted in accordance with the principles set forth in the Declaration of Helsinki. 


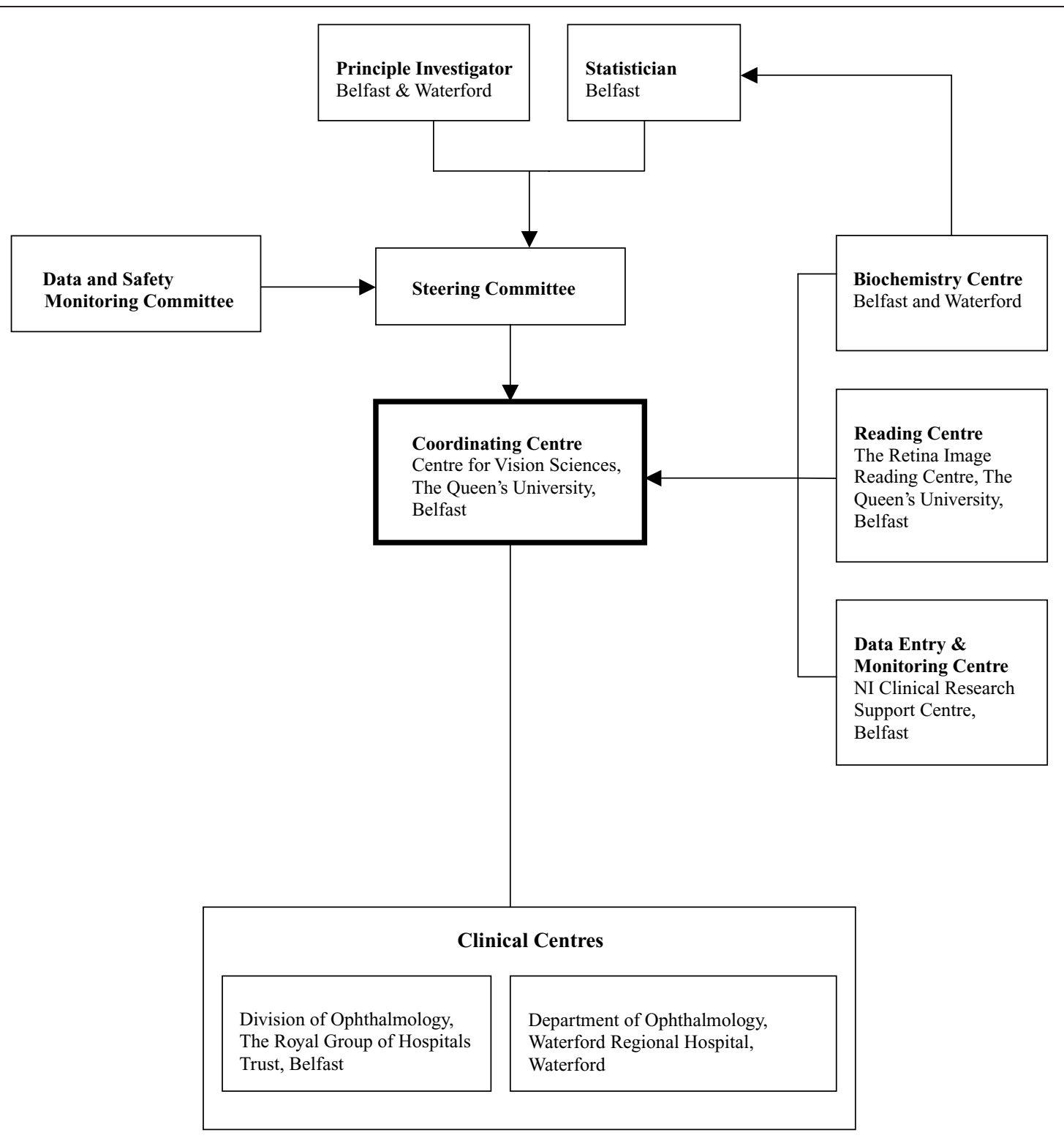

Figure 1. The CARMA Organization.

\section{CLINICAL EXAMINATION}

The clinical assessment was conducted by either an ophthalmologist or optometrist, supported by a study nurse, and consisted of ophthalmic and systemic examination.

\section{Ophthalmic examination}

\section{Distance visual acuity}

Best-corrected DVA is determined using retro-illuminated Early Treatment Diabetic Retinopathy Study (ETDRS, Precision Vision, USA) charts viewed at 4-m distance with room lights turned off. After refracting the participant with ETDRS "R" chart, using a combination of objective and subjective refraction, the best corrected VA for each eye is measured with the standard ETDRS protocol. ${ }^{34,35}$ If VA is worse than $0.8 \log$ MAR, i.e. fewer than 15 letters are read correctly at 4-m distance, measurement of VA is repeated at 1-m distance. During recording of vision on a score-sheet, each letter read correctly by the participant is encircled whereas those letters identified incorrectly are crossed.

\section{Photopic interferometric acuity (Retinal acuity)}

Interferometry is performed under photopic conditions using a white light interferometer (Lotmar Visometer, Bern, Switzerland). ${ }^{36}$ A total of 20 readings of the threshold spatial frequency are made ( 5 for each orientation with the stimuli presented randomly) and the mean value calculated. The Purkinje image of the green light on the cornea is monitored throughout 
Table 3. Inclusion and Exclusion Criteria for the CARMA Study

Inclusion criteria

- Age 50 years or older.

- Either gender.

- Able to give informed consent, make the required study visits, and adhere to the protocol.

- Fundus characteristics:

Group 1-Participants with CNV or central GA in one eye, and any level of AMD in the fellow eye with a best-corrected VA of 0.3 logMAR or better.

Group 2-Participants with early AMD features of sufficient severity in at least one eye. AMD features of sufficient severity are soft distinct or indistinct drusen $>20$ or if fewer than 20 soft drusen, focal hyper pigmentation must be present. Best-corrected VA in the study eye must be 0.3 logMAR (Snellen equivalent: 6/12) or better. Both eyes of a participant may be eligible for inclusion into the study.

Exclusion Criteria

Systemic conditions

- History of any life threatening or terminal medical condition, such as malignant disorders and cerebrovascular accidents, which may prevent follow-up visits.

- A history of major psychiatric illness and/or poor cognitive function precluding the giving of informed consent.

- History of severe gastro-intestinal disease and/or malabsorption that may hinder the absorption of active ingredients from the gastro-intestinal tract.

- Known allergy to one of the active ingredients, or other excipients in the study preparation.

Ophthalmic conditions

- History of ophthalmic disease in the study eye, such as glaucoma and diabetic maculopathy.

- History of retinal laser therapy in the proposed study eye.

Supplement use

- Prior and concurrent use of non-study preparation supplements containing vitamin $\mathrm{C}$ and $\mathrm{E}, \mathrm{Zn}, \mathrm{L}$ and $\mathrm{Z}$.

*Participants consuming vitamins or natural/herbal remedies that are not constituents of the CARMA preparation are eligible to participate in the CARMA Study. The screening/baseline examination is conducted after a washout period of 3 months.

the test to ensure correct alignment was maintained, as this can influence target visibility.

\section{Contrast sensitivity}

Contrast sensitivity is measured for each eye using PelliRobson contrast sensitivity charts (Clement Clarke International, Harlow, UK) viewed at a 1-m distance, and with +0.5 diopter addition to the participant's refractive correction. The right and left eye is tested with the chart having $\mathrm{V}, \mathrm{R}, \mathrm{S}$ and $\mathrm{H}, \mathrm{S}, \mathrm{Z}$ as the first triplet, respectively. Care is taken to ensure uniform illumination of the chart, with luminance ranging from 60 to 120 foot-candles, and to conceal the chart from viewing until the participant is ready for testing. Recording of contrast sensitivity on a score-sheet is done in a manner similar to the recording of VA.

\section{Hyperacuity}

Hyperacuity is assessed using the recently developed shape discrimination charts consisting of radial frequency patterns as the test targets. ${ }^{37}$ The test is performed under room lighting conditions at standard viewing distance $(38 \mathrm{~cm})$ with reading correction in place. As with contrast sensitivity, the participant is encouraged to guess the deformed circle when the task is perceived as being too difficult.

\section{Anterior and posterior segment examination}

A comprehensive slit lamp biomicroscopic examination is performed after pupillary dilation. Anterior segment examination consists of a standardized detailed examination of the cornea, the anterior chamber, and the lens. Lenticular opacities are graded using Lens Opacities Classification Systems II. ${ }^{38}$ Fundus examination involves identification of AMD lesions, such as drusen (type, number, and location) and/or pigmentary changes (type, location, and nature). Participants requiring intervention for recent evidence of neovascular disease and any detected eye diseases (such as advanced cataract) are referred to the principal investigator at the site for further management.

\section{Macular pigment measurement}

Macular pigment is measured using Raman spectroscopy, which is an objective and non-invasive technique of measuring MP in vivo ${ }^{39,40}$ Detailed information of principle, apparatus, and procedure, has been described elsewhere. ${ }^{41}$

\section{Anterior and posterior segment photography}

The anterior and posterior segments are photographed using a digital Topcon Fundus Camera (Topcon TRC-50EX, Topcon, Newbury, UK). Anterior segment images are graded for iris color and lenticular opacities. The posterior segment images, which consist of two non-simultaneous stereoscopic pairs centered on the macula with a 35-degree field, are obtained after full pupillary dilation. All images are stored as uncompressed tagged image files, copied to compact discs and send to the grading center, on a monthly basis.

\section{Reading center fundus grading}

Stereoscopically captured color retinal images are classified and graded in a masked fashion by any one of the five graders (who have completed the grader training and concordance exercises for the CARMA Study) using definitions of Wisconsin Age-Related Maculopathy Grading System (WARMGS) for early and late AMD features. ${ }^{42}$ The training for graders was provided in-house using a structured training program along with external qualified photo graders from the Wisconsin Fundus Photograph Reading Centre and the Complications of AMD Treatment Reading Centre. Concordance exercises were carried out between Reading Centre Graders and representatives from the above institutions. Ongoing quality assurance assessments demonstrate that our inter-observer agreement values are 
comparable to past studies ${ }^{43}$ including one from our Reading Centre. $^{44}$

Fundus photographs are sent to the senior grader and Reading Centre Clinician for adjudication in cases where a grader is not $100 \%$ confident of the AMD features to be graded. Ten percent of gradings are randomly selected to be re-graded, and any discrepancies are arbitrated by the Reading Centre clinician. As the CARMA Study is a longitudinal study, changes in morphological features of AMD are monitored over time, and in any fundus photograph where progression or regression to a different stage of AMD is deemed to have occurred, the images are routinely checked by a senior grader.

\section{General examination}

The general physical examination consisted of measurement of ambulatory blood pressure and body mass index.

\section{Blood sample collection}

For each participant, a peripheral venous blood sample of $20 \mathrm{ml}$ is obtained. The participants are non-fasting; however, they are requested not to take study medication, fruit or fruit juices on the day of blood sample collection. Blood samples are centrifuged within four hours of collection (3000 rpm, 15 minutes), and the resultant serum and plasma layers are aliquoted into 12 micro centrifuge tubes and stored at -70 degree Celsius (serum tubes: 4; plasma tubes: 4; vitamin C tubes: 2; DNA tubes: 2). The two micro centrifuge tubes, which are used for estimation of vitamin C, consist of $100 \mu \mathrm{l}$ plasma stabilized with $900 \mu \mathrm{l}$ of 5\% metaphosphoric acid (which is freshly prepared weekly). Buffy coats (collected at a single visit only) are stored in 2 micro centrifuge tubes for future genetic studies. Serum samples packed in dry ice are shipped to the central laboratory on a 3monthly basis where they are stored at -70 degree Celsius until analyzed by reverse-phase high pressure liquid chromatography.

\section{Questionnaires}

The general life-style and food frequency questionnaire are administered during the baseline visit. The former gathers information relating to socio-demographic details, smoking habits, past medical history, use of aspirin and other prescription/or over-the-counter medications, and in women a reproductive history including hormone replacement therapy is included.

A validated semi-quantitative food frequency questionnaire evaluates the dietary intake for each participant for the 12 months immediately preceding the date of randomization(Willet W. Nutritional Epidemiology. Oxford: Oxford University Press, 1990). The questionnaire comprises a list of 147 food items which are divided into 12 sections: meat, fish,bread and savory biscuits, cereals, rice and pasta, dairy products, fats and dressings, sweets and snacks, soups and sauces, drinks, fruits, vegetables. The options for frequency of consumption are provided for each food item, and quantified in appropriate units with reference to standard portion sizes. At baseline, the dietary intake of $\mathrm{L}$ and $\mathrm{Z}$ will be assessed in both the intervention and in the control group.

\section{Randomization}

Each participant enrolled in the CARMA Study is allocated a unique number, which determines treatment allocation according to the computerized randomization database. This unique number exists on the identification label of each studypreparation box. The masked study-preparation boxes are kept in the hospital pharmacy, and released in a sequential manner by the pharmacist on randomization of each participant, beginning with the first in the numerical series assigned to each clinical center. The participants are advised to take 1 tablet twice daily with a meal.

The CARMA Study is strictly a double-masked clinical trial in that neither the CARMA participants nor the study staff, including the study investigator, are aware of the nature of study preparation allocated to the participants. To ensure masking, the study-preparation boxes are labeled with pre-assigned numbers at the site of manufacturing, and then shipped to both clinical centers for distribution. A single pharmacist involved with manufacturing of the study preparation holds the key to randomization of the CARMA supplements.

\section{Summary of the baseline data}

In the CARMA Study, we have enrolled 433 participants, with $237(55 \%)$ and $196(45 \%)$ in groups 1 and 2, respectively. The mean age of the study population $( \pm \mathrm{SD})$ was $75.93 \pm 7.74$ years, ranging between 50 and 95 years. Of the 433 participants, $248(57 \%)$ were females and $185(43 \%)$ were males.

At baseline visit, the mean $( \pm \mathrm{SD}) \log$ MAR VA was comparable in the two groups [Group $1=0.08 \pm 0.12$ (range: -0.3 to 0.3 ); Group $2=0.08 \pm 0.14$ (range: -0.2 to 0.5 )]. Similarly, there was no significant difference in total number of logMAR letters recognized by the participants belonging to the two groups [Group 1: $=50.80 \pm 6.43$ (range: 36 to 67 ); Group $2=50.60$ \pm 7.44 (range: 29 to 65 )].

\section{Follow-up visits}

Follow-up visits occur at 6 monthly intervals, with telephone contact occurring at 2 -weeks and at 3 -months. The scheduling of the follow-up visit at the study center is determined by the date of randomization \pm 7 days. At repeat study visits, the clinical and ophthalmic assessments are repeated (Fig. 2). Information on intercurrent illness, changes in regular medication and potential adverse events, are recorded on a standardized proforma. At the conclusion of the study visit, a further 6-month supply of the study preparation is provided, and the importance of adherence with the study protocol is re-emphasized to participants.

The compliance with study-preparation is assessed as follows: self-reporting the precise number of left-over tablets by 2-weekly and 3-monthly telephone contacts; tablet count of the returned study-preparation box at each follow-up visit. 


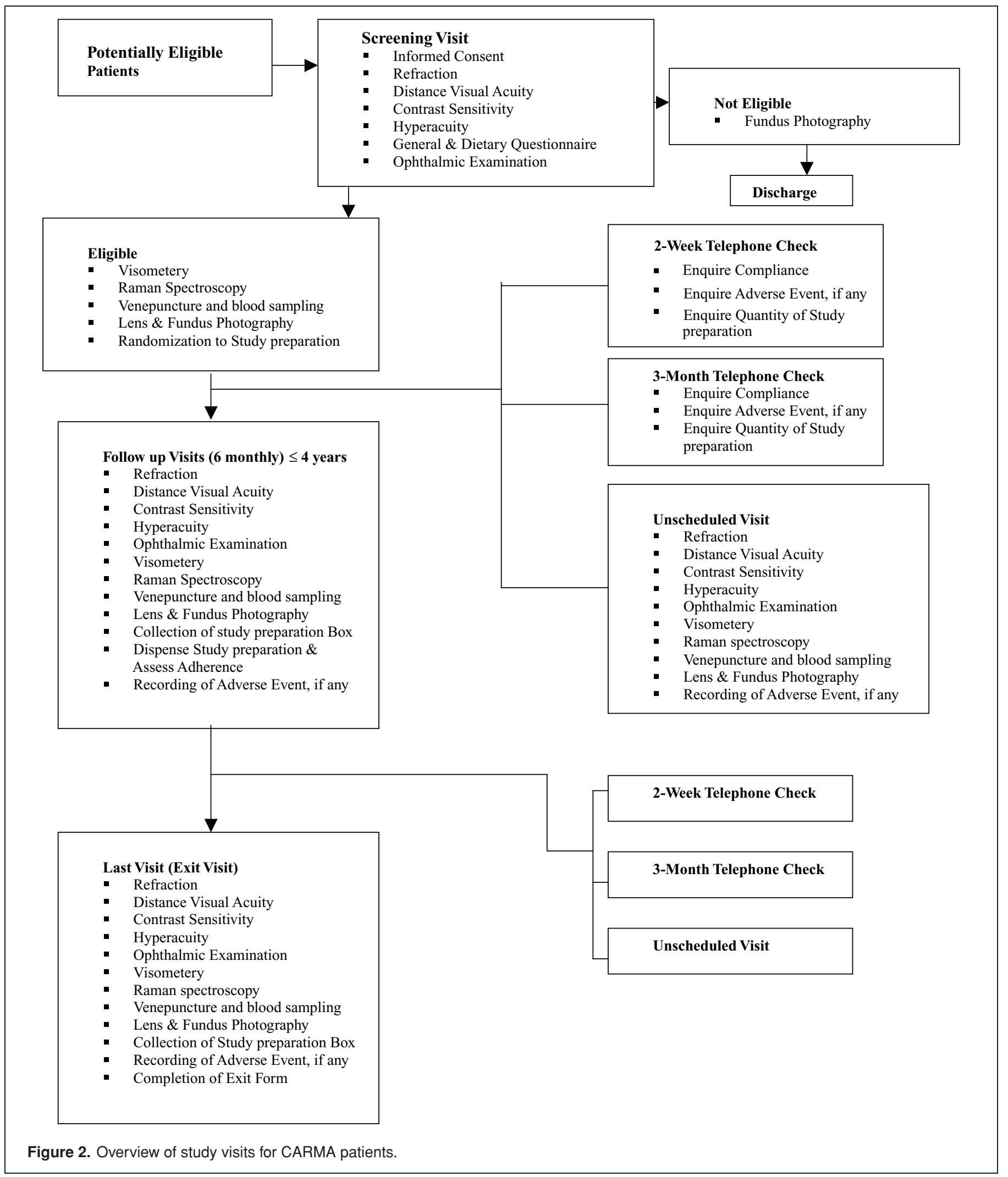




\section{Withdrawal and termination of followup visits}

Participants are encouraged to adhere to the study protocol. However, where a participant wishes to discontinue his/her participation in the CARMA Study, whether voluntarily or because of intercurrent medical indication (such as development of severe systemic illness which preclude the participant from continuing the study preparation or attending the follow-up visits), an exit visit is scheduled. During this visit, a clinical and ophthalmological assessment with photography is undertaken and an exit form (stating the reason for his/her premature exit from the study) is completed. Participants who are withdrawn from the study are not replaced, and their identification numbers are not reassigned.

\section{Unscheduled visits}

Unscheduled visits are conducted at the discretion of the investigator, and the information collected during such visits is recorded on the Unscheduled Visit Forms. If a participant wishes to discontinue at an unscheduled visit, the exit form is also completed.

\section{Study preparation}

The study preparation, manufactured by Bausch and Lomb (Berlin), consists of either placebo or L $(6 \mathrm{mg}), \mathrm{Z}(0.3 \mathrm{mg})$, and co-antioxidants (vitamin E: $7.5 \mathrm{mg}$; vitamin C: $75 \mathrm{mg}$; zinc: $10 \mathrm{mg}$; copper: $0.2 \mathrm{mg}$ ) as main ingredients in a tablet. Beta-carotene, a carotenoid with antioxidant properties, is not included in the study preparation, as there is an increased risk of lung cancer in smokers who take supplements containing beta-carotene. ${ }^{45,46}$ The placebo preparation consists of cellulose microcrystalline, lactose, and magnesium stearate, and are indistinguishable from the intervention preparation in size, color, smell, and taste. The study preparation is produced according to Good Medical Practice as laid down in relevant European directives, and currently has a shelf life of 24 months.

All the main ingredients of the study preparation belong to the vitamin/micronutrient group, which are found in a typical diet. Therefore, it is expected that the study preparation should be well tolerated, and no side effects are anticipated when used according to the study protocol, although mild gastro-intestinal irritations may occur in a small number of participants. Allergic reactions against the main ingredients or other excipients of the study preparation may occur.

\section{Safety of lutein and zeaxanthin}

Three studies have investigated L toxicity in animal models, and the data from these studies demonstrate that there have been no adverse clinical or histopathological changes suggestive of toxicity, following administration of high doses of L (up to 20 $\mathrm{mg} \mathrm{L} / \mathrm{kg}$ body weight/day for 52 weeks) ${ }^{47-49}$ Furthermore, a number of human interventional studies, involving supplementation with high doses of $\mathrm{L}$ for extended periods of time, have failed to demonstrate any toxicity in ocular and non-ocular tissues ( $20 \mathrm{mg}$ of $\mathrm{L}$ per day for 6 months). ${ }^{50-53}$ The only side effect reported as a result of $\mathrm{L}$ supplementation in humans has been carotenedermia, a harmless and reversible cutaneous hyperpigmentation, which is not associated with any specific adverse effect on health. ${ }^{54-56}$

\section{Adverse events}

If an adverse event is detected or reported at any stage in the study, an adverse event form is completed within five days of the investigator being made aware of such an event. This form contains information on the event, with particular attention directed towards the date of onset, description of event, severity, possible explanation, date of resolution (if any), relationship to the study preparation, and the action taken with the study preparation. If an adverse event is believed to be attributable to the study preparation (such as an allergic reaction), the participant is advised to discontinue the study preparation, immediately and permanently.

Every attempt is made to classify the event into a severe adverse event or an adverse event, this categorization being consistent with the ICH guidelines (Definitions and Standards for Expedited Reporting, 2003). In the event of death, the medical records, including the details of death certification, are obtained, where available, to allow determination of the cause of death and its relationship, if any, with the study preparation. All adverse events are reported to the health authorities concerned. It is mandatory to report serious adverse events within a timeframe of 7 days of the principal investigator being notified of such an event.

\section{Outcome measures}

The primary outcome variable is a change in the DVA of the study eye at 12 months of antioxidant supplementation. The level of significance for a change in DVA was set at 0.05 . The secondary outcome measures include retinal visual acuity, morphological progression of AMD, MP levels, and serum levels of antioxidants. Morphological status of AMD is based on detailed grading of stereoscopic color fundus photographs.

The development of CNV or central GA is considered end stage disease, and if reached, will be handled as follows: In group 1, participants will exit the study if CNV or central GA develops in the sole study eye. In group 2, where both eyes are study eyes, the development of CNV or central GA in one eye only is not a reason to exit the study, and the participant remains in the study unless the second eye also develops end stage disease. Participants who develop CNV during the course of study are referred to the principle investigators for appropriate management.

\section{DATA ANALYSIS}

In the present study, the main outcome DVA (expressed in units which are the logarithm of the minimum angle of resolution) will be measured as the average change in DVA across the number of eyes recruited to the study. The weights assigned to each participant will be obtained from 
the formula suggested in the study protocol, and is expanded below.

\section{Handling of data from Group 2 participants}

Several papers mention the lack of statistical independence when dealing with outcomes in paired organs. This is a particular problem in ophthalmology as the outcomes measured in both eyes of an individual are likely to be more closely related than outcomes measured on eyes from different individuals. Earlier reports have examined dichotomous outcomes, and so the solution becomes a particular application of intra-class correlation. In this study, the main outcome, DVA, will be treated as continuously distributed. One possible solution is simply to use data from one eye only from participants in whom both eyes fit the entry criteria. This method imposes a strict one eye per participant regime. The disadvantage of this approach is that it does not utilize all readily available data, and is therefore cost inefficient. Collecting all data and treating them as independent outcomes can also be flawed for reasons stated above. A compromise solution is suggested that follows parallel arguments to that for intra-class correlation. ${ }^{57}$

Group 1 participants will only have one study eye (the fellow eye having already been lost to exudative AMD). Let the variance associated with change in DVA in this group be $\sigma_{1}^{2}$. Group 2 participants may have both eyes eligible for the study, and thus have two components of variance that can be estimated. That between participants will be denoted $\sigma_{2 \mathrm{~B}}^{2}$ and that between eyes within the same participant be $\sigma_{2 \mathrm{~W}}^{2}$. In practice the study will yield some large sample estimators $\mathrm{s}_{1}^{2}, \mathrm{~s}_{2 \mathrm{~B}}^{2}$ and $\mathrm{s}_{2 \mathrm{~W}}^{2}$. A reasonable prior expectation might be for homogeneity of variance across all participants, where $\sigma_{1}^{2}=\sigma_{2 \mathrm{~B}}^{2}+\sigma_{2 \mathrm{~W}}^{2}$.

However, the approach suggested above will permit heterogeneity of variance between groups. It is usual, in situations of heterogeneity of variance, to weight cases by the reciprocal of the variation associated with each case. We propose, therefore, that all data be collected and the variance estimates described above calculated. Data will be aggregated to one result per participant - the sole result will stand for group 1 participants, and the mean of the two results will be applied to group 2 participants. The variance associated with group 1 participants will be $\mathrm{s}_{1}^{2}$, while that associated with the mean of group 2 participants will be $s_{2 \mathrm{~B}}^{2}+s_{2 \mathrm{~W}}^{2} / 2$. Therefore, in the main analysis, group 1 participants will be given a weight of 1 , whereas group 2 participants will be given a weight: $\mathrm{w}_{2}=\mathrm{s}_{1}^{2} /\left(\mathrm{s}_{2 \mathrm{~B}}^{2}+\mathrm{s}_{2 \mathrm{~W}}^{2} / 2\right)$.

We note that if most of the variation lies between participants, then $\mathrm{w}_{2}$ will tend to be close to 1 . Here, the second eye from group 2 participants provides very little additional information. However, if most of the variation lies within participants, then $\mathrm{w}_{2}$ will tend to be close to 2 . In this situation, the second eye from participants in group 2 contributes additional information, and we are moving towards a situation where both eyes of a participant have been entered as independent observations. It is important to note this approach is essentially weighted by reciprocal of the variation associated with each observation, which is among the most common weighting strategies.

\section{Analysis of primary outcome}

These data will be analyzed by an independent samples t-test with arm of the study as grouping variable. All tests will be performed as 2-tailed hypotheses, and a p-value of 0.05 will be regarded as statistically significant. For the purposes of analysis, eyes with extremely poor vision i.e., worse than $1.6 \log \mathrm{MAR}$ will be allocated a score of 1.7, and we do not intend to differentiate between hand movements and light perception.

\section{Adjustment for covariates}

Distance VA, as defined above at 12 months after randomization into the trial, will be regressed on DVA at baseline, with study group to which randomized as main factor, and linear adjustments allowed for variables (variable types) defined below:

- Gender (dichotomous)

- Age (continuous)

- Bilateral involvement (dichotomous)

- Smoking status (categorical)

- Eye color (categorical, but probably dichotomized)

- Use of vitamin supplements and/or diet at baseline (continuously distributed).

\section{ANALYSIS OF SECONDARY OUTCOMES}

This will depend on the type of variable. Categorical variables will be analyzed by chi-squared or Fisher's Exact tests (the latter being employed when expected counts drop close to or below 5.0). Continuously distributed variables will be analyzed by independent sample t-tests, after assessment of whether equal or unequal variances can be assumed. Adjustment for covariates will be performed when appropriate, and by logistic regression for dichotomized categorical variables, and linear regression for continuously distributed outcomes. Tests for heterogeneity of variance and parallelism will be employed to check the validity of these models. Transformations will be attempted when the usual assumptions fail.

In this study, it is not expected that many outcomes will be of an ordinal nature. However, if because of failed distributional assumptions, continuously distributed variables have to be transformed to ordinal scales, then the module for ordinal regression will be employed along with tests for parallelism. Although some secondary outcomes such as CS, like distance VA, will be assessed at the level of eye, such outcomes will be combined by the method suggested above, and the analysis will continue with the experimental unit as a single participant and using appropriate statistical methods.

\section{QUALITY CONTROL}

Various steps are taken to ensure adequate quality control for the CARMA Study. First, a comprehensive study manual, with detailed and clear instructions for carrying out the study procedures is available. Second, study staff at the two centers work to agreed protocols. Third, both centers are equipped with 
identical instruments which are calibrated to agree preset standards. Fourth, the eligibility criteria are verified by an examining ophthalmologist at the screening visit before randomization. Where eligibility is in question, adjudication by both principal investigators is used to determine the ultimate eligibility of the patient. Fifth, centralized data management facilitates scrutiny of the CRF for completeness and consistency, with queries resolved rapidly. Finally, regular monitoring and quality control is undertaken by the coordinating center.

\section{SAFETY AND DATA MONITORING COMMITTEE}

An independent DSMC scrutinizes the unblinded data for evidence of safety and efficacy every year. This committee comprises of three internationally recognized scientific and clinical members (two ophthalmologists and one physician epidemiologist), and has the power to recommend any of the following; continuation of the study uninterrupted, alteration of the study or any arm of the study, or termination of the study or any arm of the study.

\section{DISCUSSION AND EXPECTED CONTRIBUTION}

The CARMA Study is an important interventional study of antioxidant supplementation in AMD patients. Similar to AREDS II, ${ }^{58}$ the CARMA Study is a randomized, double masked, and placebo controlled clinical trial of $\mathrm{L}$ and $\mathrm{Z}$ along with co-antioxidants in AMD participants with a reasonably good sample size. However, the CARMA supplement does not include omega 3 poly-unsaturated fatty acids, which are increasingly believed to play a protective role in AMD.

Furthermore, and similar to the LAST trial, ${ }^{30}$ the CARMA Study is examining the effect of antioxidant supplementation on psychophysical visual function, such as DVA, contrast sensitivity, and retinal visual acuity. However, in the LAST trial, the study population consisted of 90 male participants with advanced AMD (atrophic) and at least one vision-degrading psychophysical abnormality. Whereas, in the CARMA Study, 248 females and 185 males with either early AMD features of sufficient severity in at least one eye or any level of AMD in one eye and advanced AMD in the fellow eye have participated. Also, the OcuPower supplement in the LAST trial consisted of a large number of nutrients in contrast to the CARMA supplement, which contains 5 major antioxidants, including $\mathrm{L}$ and $\mathrm{Z}$ that constitute MP.

The CARMA Study will provide invaluable and timely data on the potential role of antioxidants, including $\mathrm{L}$ and $\mathrm{Z}$, in delaying AMD progression. An observed beneficial effect of such intervention will complement evidence from other completed and ongoing randomized clinical trials, and inform current practice with respect to the role of dietary modification and/or supplementation in patients with AMD. The CARMA Study will also yield valuable information with respect to visual function and MP levels following supplementation with L, Z, and antioxidants in eyes with AMD. Finally, the CARMA Study will provide data on the safety and toxicity of L, Z, and antioxidant supplementation in human subjects.

\section{CARMA RESEARCH GROUP}

Anderson Roger, Psychophysics, Coleraine

Beatty Stephen, Principal investigator, Waterford

Chakravarthy Usha, Principal investigator, Belfast

Hogg Ruth, Study optometrist, Belfast

Neelam Kumari, Study clinician, Waterford

O’ Donovan Orla, Biochemist, Waterford

Stevenson Michael, Study statistician, Belfast

Young Ian, Biochemist, Belfast

\section{DATA SAFETY AND MONITORING COMMITTEE}

Professor Astrid E. Fletcher, Chair, Professor of Aging and Population Health, The London School of Hygiene and Tropical Medicine, London, UK.

Professor Frank G. Holz, Chairman, Department of Ophthalmology, University of Bonn, Germany.

Professor Fredericus J. Van Kuijk, Vice Chair for Clinical Services, Department of Ophthalmology and Visual Sciences, University of Texas, Austin, Texas, USA.

\section{ACKNOWLEDGMENT}

This study was supported by a grant from Bausch and Lomb, Berlin.

\section{REFERENCES}

1. Congdon N, O'Colmain B, Klaver CCW et al. Causes and prevalence of visual impairment among adults in the United States. Arch Ophthalmol. 2004;122:477-85.

2. Evans JR, Fletcher AE, Wormald RPL. Causes of visual impairment in people aged 75 years and older in Britain: an add-on study to the MRC Trial of Assessment and management of older people in the community. Br J Ophthalmol. 2004;88:365-70.

3. Friedman DS, O'Colmain BJ, Munoz B et al. Prevalence of agerelated macular degeneration in the United States. Arch Ophthalmol. 2004;122:564-72.

4. Hyman LG, Lilienfeld AH, Ferris FL et al. Senile macular degeneration: A case-control study. Am J Ophthalmol. 1983;118:213-22.

5. Leibowitz HM, Krueger DE, Maunder LR. The Framingham Eye Study. Surv Ophthalmol. 1980;24 (Suppl).

6. Bartlett $\mathrm{H}$, Eperjesi $\mathrm{F}$, Ali $\mathrm{A}$ et al. Risk factors associated with agerelated macular disease. Optom Practise. 2004;5:15-32.

7. Owen $C G$, Fletcher $A E$, Donoghue $M$ et al. How big is the burden of visual loss caused by age-related macular degeneration in the United Kingdom? Br J Ophthalmol. 2003;87:312-7.

8. Macular Photocoagulation Study Group. Argon laser photocoagulation for neovascular maculopathy. Five-year results from controlled clinical trials. Arch Ophthalmol. 1991;109:1109-14.

9. Treatment of Age-related Macular Degeneration with Photodynamic therapy (TAP) Study Group. Photodynamic therapy of 
subfoveal choroidal neovascularization in age-related macular degeneration with verteporfin: One-year results of 2 randomized clinical trials-TAP report No 1. Arch Ophthalmol. 1999;117:1329_ 45

10. Treatment of Age-related Macular Degeneration with Photodynamic Therapy (TAP) Study Group. Photodynamic therapy of subfoveal choroidal neovascularization in age-related macular degeneration with verteporfin: Two-year results of 2 randomized clinical trials-TAP report 2. Arch Ophthalmol. 2001;119:198207.

11. Verteporfin In Photodynamic Therapy Study Group. Verteporfin therapy of subfoveal choroidal neovascularization in age-related macular degeneration: Two-year results of a randomized clinical trial including lesions with occult with no classic choroidal neovascularization-Verteporfin In Photodynamic therapy report 2. Am J Ophthalmol. 2001;131:541-60.

12. Gragoudas ES, Adamis AP, Cunningham ETJ et al. Pegaptanib for neovascular age-related macular degeneration. N Engl J Med. 2004;351:2805-16.

13. VEGF Inhibition Study in Ocular Neovascularization (V.I.S.I.O.N) Clinical Trial Group. Year 2 efficacy results of 2 randomized controlled clinical trials of pegaptanib for neovascular age-related macular degeneration. Ophthalmology. 2006;113:1508-21.

14. Cahill MT, Banks AD, Stinnett SS et al. Vision-related quality of life in patients with bilateral severe age-related macular degeneration. Ophthalmology. 2005;112:152-8.

15. Davis CA, Lovie-Kitchin JE, Thompson B. Psychosocial adjustment to age-related macular degeneration. J Vis Impair Blindness. 1995;89:16-27.

16. DeCarlo DK, Scilley K, Wells $\mathrm{J}$ et al. Driving habits and healthrelated quality of life in patients with age-related maculopathy. $O p$ tom Vision Sci. 2003;80:207-13.

17. Lamoureux EL, Hassell JB, Keeffe JE. The determinants of participation in activities of daily living in people with impaired vision. Am J Ophthalmol. 2004;137:265-70.

18. Stevenson MR, Hart PM, Montgomery AM et al. Reduced vision in older adults with age-related macular degeneration interferes with ability to care for self and impairs role as carer. $\mathrm{Br} J$ Ophthalmol .2004;88:1125-30.

19. Williams RA, Brody BL, Thomas RG et al. The psychosocial impact of macular degeneration. Arch Ophthalmol. 1998;116:514-20.

20. Beatty S, Boulton ME, Henson DB. Macular pigment and agerelated macular degeneration. Br J Ophthalmol. 1999;83:86777

21. Landrum JT, Bone RA, Kilburn MD. The macular pigment: A possible role in protection from age-related macular degeneration. Adv Pharm. 1997;38:537-56.

22. Bone RA, Landrum JT, Tarsis SL. Preliminary identification of the human macular pigment. Vision Res. 1985;25:1531-5.

23. Snodderly DM, Handelman GJ, Adler AJ. Distribution of individual macular pigment carotenoids in central retina of macaque and squirrel monkeys. Invest Ophthalmol Vis Sci. 1991;32:268-79.

24. Johnson EJ, Hammond BR, Yeum KJ. Relation among serum and tissue concentrations of lutein and zeaxanthin and macular pigment density. Am J Clin Nutr. 2000;71:1555-62.

25. Age-Related Eye Disease Study Group. A randomized, placebocontrolled clinical trial of high-dose supplementation with vitamins $C$ and $E$, beta carotene, and zinc for age-related macular degeneration and visual loss. Arch Ophthalmol. 2001;119:141736.

26. Barlett HE, Eperjesi F. Effect of lutein and anti-oxidant dietary supplementation on contrast sensitivity in age-related macular disease: A randomized controlled trial. Eur J Clin Nutr. 2007;31.

27. Massacesi KR, Faletra R, Gerosa F. The effect of oral supplementation of macular carotenoids (lutein and zeaxanthin) on the prevention of age-related macular degeneration: A 18 months of follow up study. Ass Res Vis Ophthalmol. 2001;42:234.
28. Olmedilla B, Granado F, Blanco I et al. Lutein in patients with cataract and age-related macular degeneration: A longterm supplementation study. J Sci Food Agr. 2001;81:9049.

29. Richer S. ARMD-pilot (case-series) environmental intervention data. J Am Optom Assoc. 1999;70:24-36.

30. Richer S, Stiles W, Statkute L. Double-masked, placebo-controlled, randomized trial of lutein and antioxidant supplementation in the intervention of atrophic age-related macular degeneration: The Veterans LAST study (Lutein Antioxidant Supplementation Trial). Optometry. 2004;75:216-30.

31. Chong EW-T, Wong TY, Kreis AJ et al. Dietary antioxidants and primary prevention of age-related macular degeneration: Systematic review and meta-analysis. BMJ. 2007.

32. Beatty $\mathrm{S}$, Koh $\mathrm{HH}$, Henson $\mathrm{D}$ et al. The role of oxidative stress in the pathogenesis of age-related macular degeneration. Surv Ophthalmol.2000;45:115-34.

33. Augood C, Fletcher A, Bentham G. Methods for a population-based study of the prevalence of and risk factors for age-related maculopathy and macular degeneration in elderly European populations: The EUREYE Study. Ophthalmic Epidemiol. 2004;11:11729.

34. Ferris FL, Kassoff A, Bresnick GH, Bailey I. New visual acuity charts for clinical research. Am J Ophthalmol. 1982;94:91-96.

35. Ferris FL, Kassoff A, Bresnick G. New visual acuity charts for clinical research. Am J Ophthalmol. 1982;94:91-6.

36. Grignolo FM, Moscone F, Carenini-Boles A. Assessment of malingerer's visual acuity by Lotmar's visometer test. Ann Ophthalmol. 1988;20:335-9.

37. Wang $Y Z$, Wilson E, Locke KG et al. Shape discrimination in age-related macular degeneration. Invest Ophthalmol Vis Sci .2002;43:2055-62.

38. Chylack LT, Leske CM, McCarthy D. Lens Opacities Classification System II. Arch Ophthalmol. 1989;107:991-7.

39. Bernstein PS, Yoshida MD, Katz NB. Raman detection of macular carotenoid pigments in intact human retina. Invest Ophthalmol Vis Sci. 1998;39:2003-11.

40. Ermakov IV, McClane RW, Gellermann W. Resonant Raman detection of macular pigment levels in the living human retina. Opt Lett. 2001;26:202-4.

41. Neelam K, O'Gorman N, Nolan JM et al. Measurement of macular pigment: Raman spectroscopy versus heterochromatic flicker photometry. Invest Ophthalmol Vis Sci. 2005;46:102332

42. Klein R, Davis MD, Magli YL. The Wisconsin age-related maculopathy grading system. Ophthalmology. 1991;98:112834.

43. Scholl HPN, Peto T, Dandekar SS et al. Interobserver and intraobserver variability in grading lesions of age-related maculopathy and macular degeneration. Graefe's Arch Clin Exp Ophthalmol .2003;241:39-47.

44. Van Leeuwen R, Chakravarthy U, Vingerling JR et al. Grading of age-related maculopathy for epidemiological studies: Is digital imaging as good as 35-mm film? Ophthalmology. 2003;110:15404.

45. Omenn GS, Goodman GE, Thornquist MD. Effects of a combination of beta carotene and vitamin A on lung cancer and cardiovascular disease. N Engl J Med. 1996;334:1150-5.

46. The Alpha-Tocopherol BCCPSG. The effect of vitamin E and beta carotene on the incidence of lung cancer and other cancers in male smokers. N Engl J Med. 1994;330:1029-35.

47. Goralczeyk R, Barker F, Froescheis $\mathrm{O}$ et al. Ocular safety of lutein and zeaxanthin in a long term study in Cynomolgous monkeys. Assoc Res Vision Ophthalmol. 2002;43:2546.

48. Jenkins MY, Mitchell GV, Grundel E. Natural tocopherols in a dietary supplement of lutein affect tissue distribution of tocopherols in young rats. Nutr Cancer. 2000;37:207-14. 
49. Kruger CL, Murphy M, DeFreitus Z. An innovative approach to the determination of safety for a dietary ingredient derived from a new source: Case study using crystalline lutein product. Food ChemToxicol. 2002;40:1535-49.

50. Aleman TS, Duncan JL, Bieber ML. Macular pigment and lutein supplementation in retinitis pigmentosa and Usher syndrome. Invest Ophthalmol Vis Sci. 2001;42:1873-81.

51. Dagnelie G, Zorge IS, McDonald TM. Lutein improves visual function in some patients with retinal degeneration; A pilot study via the internet. Optometry. 2000;71:147-64.

52. Duncan IL, Aleman TS, Gardner LM et al. Macular pigment and lutein supplementation in choroideremia. Exp Eye Res. 2002;74:371-81.

53. Landrum JT, Bone RA, Joa H. A one year study of the macular pigment:The effect of 140 days of a lutein supplement. Exp Eye Res. 1997;65:57-62.
54. Granado F, Olmedilla B, Gil-Martinez E et al. Lutein ester in serum after lutein supplementation in human subjects. Br J Nutr. 1998;80:445-9.

55. Olmedilla B, Orarado F, Gil-Martinez E et al. Supplementation with lutein (4 months) and alpha-tocopherol (2 months), in separate or combined oral doses, in control men. Cancer Lett. 1997;114:17981.

56. Olmedilla B, Granado F, Southon S et al. A European multicentre, placebo-controlled supplementation study with alpha-tocopherol, carotene-rich palm oil, lutein or lycopene: Analysis of serum responses. Clin Sci (Lond.) 2002;102:447-56.

57. Rosner B. Statistical methods in ophthalmology: An adjustment for the intraclass correlation between eyes. Biometrics. 1982;38:10514.

58. Chew, E. Age-related eye disease study 2 protocol. 2006. Unpublished. 